\title{
3 Research Soure \\ Identification of lysine acetylome of oral squamous cell carcinoma by label-free quantitative proteomics
}

Jingjing Dong

Shenzhen People's Hospital

Jingquan He

Shenzhen People's Hospital

Zeyu Zhang

Jinan University First Affiliated Hospital

Wei Zhang

Shenzhen People's Hospital

Yixi Li

Jinan University First Affiliated Hospital

Hongliang Xie

Shenzhen People's Hospital

Wenxin Zuo

Shenzhen People's Hospital

Jianming Tang

Shenzhen People's Hospital

Zhipeng Zeng

Shenzhen People's Hospital

Wanxia Cai

Shenzhen People's Hospital

Liusheng Lai

People's Liberation Army Joint Logistic Support Force 924th Hospital: 181st Hospital of Chinese

People's Liberation Army

Manhua Yun

Jinan University First Affiliated Hospital

Lingjun Shen

Jinan University First Affiliated Hospital

Lianghong Yin

Jinan University First Affiliated Hospital

Donge Tang

Shenzhen People's Hospital

Yong Dai ( $\nabla$ daiyong@mail.sustech.edu.cn )

Shenzhen People's Hospital https://orcid.org/0000-0002-6840-9158 


\section{Research Article}

Keywords: Oral Squamous Cell Carcinoma, Oral Cancer, Head and Neck cancer, Lysine Acetylation, Posttranslational Modification, mRNA Splicing, Ribosome Pathway

Posted Date: August 13th, 2021

DOl: https://doi.org/10.21203/rs.3.rs-752350/v1

License: (c) (1) This work is licensed under a Creative Commons Attribution 4.0 International License. Read Full License 


\section{Abstract \\ Background}

Lysine acetylation (Kac) favors gene transcription and activates various genes involved in the regulation of oncogenesis, whereas the acetylation profiling of oral squamous cell carcinoma (OSCC) is unknown. We performed lysine acetylation analyses to achieve a comprehensive profile and revealed the specific pathogenesis in patients with OSCC.

\section{Methods}

Liquid chromatography - tandem mass spectrometry (LC-MS/MS) was utilized to investigate lysine acetylation features of tumor tissues and adjacent normal tissues from 9 patients with OCSS.

\section{Results}

Among the upregulated different acetylation proteins (DAPs), the biological process of GO analysis was closely related to cellular response to regulation of apoptotic process, and regulation of programmed cell death. KEGG enrichment analysis was associated with HIF-1 signaling pathway, ferroptosis, and JAKSTAT signaling pathway. In PPI network, seven differently Kac proteins (SRSF1, HNPNPM, PRPF8, DHX9, DHX15, RBMX, SNRPG) in MCODE1 and the top 30 hub gene involved in mRNA splicing process and spliceosome pathway. Six differently Kac modified proteins of RPS15A, RPL11, RPS11, RPS3, RPL24, RPL19 in MCODE1 was enriched in ribosome pathway, particular lower expression of RPS3, RPL24 and RPL19 were related to the overall survival of OSCC.

\section{Conclusion}

This study contributes a foundation for understanding the functions of Kac modification in OSCC and investigates lysine acetylation on proteins involved in ribosome pathway, particularly the ones that acted as hub genes and related to the OSCC survival, which may be a potential therapeutic direction of OSCC in the future.

\section{Background}

Oral squamous cell carcinoma (OSCC) arising from oral mucosal epithelium is the most common type of head and neck malignant tumor. It is the eighth most common type of cancer around the world [1]. OSCC has the characteristics of a high morbidity and mortality rate. It is known as a topically invasive tumor and has a recurrence rate of $18-76 \%$ even if appropriate treatment is performed[2]. Early diagnosis and effective therapies for OSCC are urgently needed. We utilise bioinformatics analyses to explore the pathogenesis and potential therapeutic direction of OSCC. 
With the wide application of sequencing technology, more than 400 types of posttranslational modification (PTMs) have been identified, including the classic acetylation and novel crotonylation and 2hydroxyisobutyrylation, etc.[3-5]. As reversibly chemical modifications of a target protein, PTMs are closely associated with numerous biological processes, including gene transcription, cell apoptosis, and cancer cell proliferation [6-8]. It has been reported lysine acetylation is closely related to cancer[8]. Acetylation of lysine is the most important PTM on histone and has a tight relationship with transcription, chromatin architecture, and DNA repair. It is reported that histone acetylation neutralizes the positive charge of lysine residues, promotes relaxation of the chromatin conformation favoring gene transcription and activates a variety of genes involved in the regulation of oncogenesis $[9,10]$. Chromatin acetylation induces cellular differentiation, restricts cellular transformation[11], and promotes cell proliferation and invasion in OSCC[12]. Histone deacetylase (HDAC) inhibitors induce to decreased number of cancer stem cells by up-regulating histone acetylation state and results in inhibition of clonogenic sphere formation[13]. It is known that HDAC inhibitors are the first kind of epigenetic-related drug that have great potential in cancer treatment, especially in anti-tumor treatment in head and neck cancer [14].

Though the lysine acetylation of histones is closely related to the development of OCSS, the acetylome atlases in OCSS and adjacent normal tissues are unknown, which hampers the understanding of acetylation role in OCSS pathology. Our study performed liquid LC-MS/MS methods to investigate lysine acetylation features in OCSS tissues and adjacent normal tissues.

\section{Methods}

\section{Sample Preparation}

Surgical tissue samples were obtained from 9 patients with OSCC diagnosed at Shenzhen People's Hospital from 2019 to 2020 . The tumor tissues were taken from the surgical resection of OSCC primary tumors. The paired samples were taken from adjacent normal tissues. Nine paired healthy and OSCC tissue samples were immediately frozen at $-196^{\circ} \mathrm{C}$ and then stored at $-80^{\circ} \mathrm{C}$. Informed consent was signed from each participant. The study was agreed by the Clinical Research Ethics Committee of the Shenzhen People's Hospital.

\section{Protein Extraction and Trypsin Digestion}

The method to extract the protein and was trypsin digestion referenced to our previous research[15]. Briefly, every tissue was grinded into cell powder and mixed with four volumes of lysis buffer (8 $\mathrm{M}$ urea, 1\% Triton X-100, $3 \mu \mathrm{M}$ TSA, 50 mM NAM, and 1\% Protease Inhibitor). Each protein sample was broken by ultrasonic exposure thrice (Scientz). Protein concentration was determined according to the manufacturer's instructions. Before trypsinization, all protein samples were diluted for to a final urea concentration less than $2 \mathrm{M}$. Then the sample was trypsinized for the first digestion overnight and second digestion with $4 \mathrm{~h}$. 
After trypsin digestion, the peptide was performed by desalination with Strata X C18 SPE column (Phenomenex, Torrance, CA) and then vacuum drying. Subsequently, reconstituted the peptides and processed with a TMT label.

\section{Affinity Enrichment, LC-MS/MS Analysis and Database Search}

Before Kac enrichment, anti-Kac beads (PTM Bio) were washed with PBS twice. Dissolved the tryptic peptides in IP solution (100 mM NaCl, 50 mM Tris-HCl, 1 mM EDTA, 0.5\% NP-40, pH 8.0) and incubated with antibody beads by gentle shaking at $4^{\circ} \mathrm{C}$ overnight. Washed the beads with NETN buffer four times and with ddH2O twice. Remove the bound peptides from the beads with $0.1 \%$ trifluoroacetic acid for LCMS/MS analysis. The methods and steps of LC-MS/MS analysis and database search referred to our previous research [15].

\section{Bioinformatic Analyses}

A quantitative Kac-proteomic method to examine lysine acetylation modification degree between the OSCC tissues and paired adjacent normal tissues were utilised, excluding the impact of the corresponding protein expression [16]. Proteins/ Kac- modified cites with a foldchange $\geq 1.50$ or $\leq 0.67$ between the OSCC tissues and paired adjacent normal tissues were considered as expression/ acetylation significant. The Clusters of Orthologous Groups (COG)/EuKaryotic Orthologous Groups (KOG) databases (http://www.ncbi.nlm.nih.gov/COG) was conducted. WOLF PSORT was utilised to predict the subcellular localization. Functional annotation enrichments of DAPs were performed by Gene Ontology (GO) analysis and Kyoto Encyclopedia of Genes and Genomes (KEGG) analysis. $P<0.05$ in Fisher's exact test and q $<0.05$ in Benjamini-Hochberg's procedure were considered as statistically significant difference. The functional protein domain descriptions were annotated based on the InterPro domain database (http://www.ebi.ac.uk/interpro/ and the protein sequence alignment. The STRING database (version 10.5) was utilised for the PPI network. A Confidence score $>0.7$ was defined as a high confidence score. Cytoscape (version 3.8.2) was used to visualize the interaction network. The top 30 hub genes were identified by CytoHubba. Molecular Complex Detection (MCODE) (version 1.5.1) was used for clusters analysis in PPI network with configuration standard as follows: Degree Cutoff = 2, MCODE scores $>5$, Node score Cutoff $=0.2, \mathrm{~K}$-Core $=2$, Max. Depth $=100$. We also analyzed the transcriptome in OSCC patients using TCGA (The Cancer Genome Atlas, https://www.cancer.gov/tcga) databases through the University of California Santa Cruz (UCSC) Xena Public Data Hub (xena.ucsc.edu). The survival analysis was performed using the survival package in $\mathrm{R}$ (3.5.0). The three-dimensional structure of modified protein constructed using SWISS MODEL(http://swissmodel.expasy.org/). A human transcription factor gene list was downloaded from Human TFDB (http://bioinfo.life.hust.edu.cn/HumanTFDB\#!/download).

\section{Results}

\section{Baseline Clinical Features}


Demographic features are summarized in Table S1. There was a total of 9 patients diagnosed with OSCC, with a median age of 50.23 years. 7 patients $(77.7 \% \%)$ had smoking history, while 2 cases $(22.2 \%)$ had a history of betel nut using. The results indicate normal complete blood count, normal serum electrolyte values, normal kidney function, and liver function.

\section{Protein Identification}

For protein expression, we found 5149 quantitative proteins from total 6658 identified proteins, in which there were 967 differential expression proteins (DEPs), including 662 proteins upregulated ( $\geq 1.5$-fold) and 305 downregulated ( $\leq 0.67$-fold) between OSCC tissues and paired adjacent normal tissues.

For protein acetylation, we identified 8388 acetylation sites in 2706 proteins. A total of 4883 acetylation sites in 1738 proteins were quantified. 2027 Kac proteins (74.9\%) had $\leq 3 \mathrm{Kac}$ sites, whereas $114 \mathrm{Kac}$ proteins (4.2\%) had $\geq 10 \mathrm{Kac}$ sites (table S2) which was more abundant than acetylation in hepatocellular carcinoma[17]. TTN, MYH2, FLNA, MYH9, MYH7, SPTAN1, AHNAK, FLNC, APOB, and MYBPC1 are the top 10 proteins with the most abundant acetylated sites. This study included 282 upregulated Kac sites ( $\geq 1.5$-fold) in 234 proteins and 235 downregulated Kac sites ( $\leq 0.67$-fold) in 162 proteins between OSCC tissues and paired adjacent normal tissues. Hierarchical clustering analysis of DEPs and DAPs is shown in Fig. 1.

For all DEPs and DAPs, we identified 132 proteins with expression difference and acetylation difference (Fig. 1d).

\section{Functional Characterization of Differentially Lysine Acetylation Proteins.}

The effects of lysine acetylation proteins were then further analyzed by Gene Ontology (GO) annotation and COG (Clusters of orthologous groups) analysis.

The function classification of differential acetylation proteins was then carried out with COG analysis. Among upregulated DAPs, we found that 27 proteins are associated with cytoskeleton (12.4\%), 22 proteins with posttranslational modification, protein turnover, chaperones $(10.1 \%)$, and 20 proteins with Translation, ribosomal structure, and biogenesis (9.2\%). Among downregulated DAPs, 23 proteins are associated with cytoskeleton (15.2\%), 21 proteins with Energy production and conversion (13.9\%), and 17 proteins with Signal transduction mechanisms (11.3\%) (Fig. 2a,2b).

The subcellular localizations of the upregulated DAPs were mainly distributed in the cytoplasm (45.49\%), nucleus (19.74\%), mitochondria (14.59\%), extracellular (6.87\%), cytoplasm, nucleus $(6.44 \%)$ and plasma membrane (3.86\%) (Fig. 2c). Among the downregulated DAPs, the subcellular localizations were mainly distributed in cytoplasm (37.89\%), mitochondria (22.36\%), extracellular (14.29\%), nucleus (11.18\%) and plasma membrane (4.97\%) (Fig. 2d).

In GO annotation, the related biological functions were established for upregulated DAPs and further separated into three classifications: cellular component (33.3\%), molecular function (22.2\%), and 
biological process (44.4\%) (Fig. 2e). Among downregulated DAPs, three classifications included cellular component (29.6\%), molecular function (25.9\%), and biological process $(44.4 \%)$. The number of DAPs involved in the three biological process groups were shown in Fig. 2e,2f.

\section{Functional Enrichment Analysis of Differentially Lysine Acetylation Proteins.}

GO enrichment analysis was performed to further understand the functions of differential lysine acetylation proteins. Among 234 upregulated DAPs, the cellular component in GO enrichment analysis significantly enriched in actin filament bundle, actomyosin, and actin cytoskeleton (Fig. 3a). Biological process was significantly enriched in cellular response to type I interferon, type I interferon signaling pathway, response to type I interferon, negative regulation of viral process, cellular response to cytokine stimulus, regulation of apoptotic process, and regulation of programmed cell death (Fig. 3b). According to molecular function enrichment classification, double-stranded RNA binding, cytoskeletal protein binding, actin filament binding, and nucleoside-triphosphatase activity were significantly enriched (Fig. 3c). Among 162 downregulated DAPs, the cellular component significantly enriched in actin cytoskeleton, contractile fiber part, and contractile fiber part (Fig. 4a). According to biological process, striatedd muscle contraction, cardiac muscle contraction, heart contraction, and positive regulation of blood circulation were highly enriched (Fig. 4b). Molecular function highly enriched in actin binding, structural constituent of muscle, ankyrin binding, and calcium ion binding (Fig. 4c).

KEGG analysis of upregulated DAPs enriched in the top 3 pathways including hepatitis C, biosynthesis of unsaturated fatty acids, fatty acid metabolism. As shown in Fig. 3d, HIF-1 signaling pathway, ferroptosis, JAK-STAT signaling pathway were also highly enriched. Among downregulated DAPs, we found that most of these proteins are involved in tyrosine metabolism, phenylalanine metabolism in KEGG analysis.

Domain enrichment of upregulated DAPs was involved in Guanylate-binding protein, C-terminal and Nterminal domain as well as Ku70/Ku80 N-terminal alpha/beta and beta-barrel domain (Fig. 3e). We found that the downregulated DAPs were significantly enriched in myosin N-terminal SH3-like domain, EF-hand domain pair, and fibronectin type III domain in domain enrichment (Fig. 4e).

\section{The Exploration of Differentially Lysine Acetylated Motifs.}

A total of 8,267 Kac peptides from all identified peptides with amino acids around the acetylated lysine from 10 amino acids upstream to 10 amino acids downstream are subjected to the Motif-X program. We identified 50 conserved motifs based on acetylated lysine. Especially, the motifs $x x x x x x x x x x \_K \_H x x x x x x K x x, x x x x x x x x x$ N_K_KAxxxxxxxxx (Motif Score $>30.00$ ) were strikingly conserved. The top 3 motifs and their abundances are as follows: $x x x x x x x x x x \_K$ _Sxxxxxxxxx (748 peptides), xxxxxxxxxx_K_Txxxxxxxxx (652 peptides), xxxxxxxxxG_K_xxxxxxxxxx (509 peptides). Overall, the present results suggested that these motifs may reveal characteristics regarding acetylation in patients with ocsS (Table S3). 


\section{PPI Network Construction and Survival Analysis of Hub Genes}

PPI network of the upregulated DAPs was illustrated in Fig. 5(a). A total of 228 nodes with 530 edges were reflected in this network system. The top 30 hub genes were identified by CytoHubba (Table S4). Of these, several proteins had abundant acetylation sites, including 15 acetylation sites on HSP90AA1, 23 acetylation sites on MYH9, 16 acetylation sites on PKM, and 12 acetylation sites on EEF2. Among the top 30 hub genes, survival analysis from the TCGA database showed that lower expression of RPS3, RPL24, RPL19, CCT8, HSP90AA1, EGFR, and ARPC2 were associated with poor prognosis (Fig. 6a-d and Figure S1 a-d). 13 functional subnet cluster were selected from the PPI network by MCODE. All the 17 proteins in cluster 1 (MCODE score 9.2) were contained in the top 30 hub genes (Fig. 5b). Biological process of GO analysis of these proteins was associated with mRNA splicing and SRP-dependent cotranslational protein targeting to membrane (Fig. 5c). KEGG enrichment analysis of cluster 1 significantly related to spliceosome and ribosome pathway (Fig. 5d). Remarkably, RPS3, RPL24, and RPL19 in cluster 1 were closely associated with OSCC prognosis.

Among the 162 downregulated DAPs, PPI network contained 159 nodes with 94 edges. The top 30 hub genes were listed in table S5. Of these, 11 proteins had more than 10 acetylation sites, especially 130 acetylation sites on TTN, 45 acetylation sites on MYH2, 27 acetylation sites on MYH7 and 24 acetylation sites on SPTAN1. Among the top 30 hub genes, the survival analysis from the TCGA database showed that low expression of EIF4A2, RPL12, MYBPC1, RPS6, ARCN1, and TMEM9 was related to poor prognosis, particularly EIF4A2 and RPL12 with a better survival curve (Fig. 7a-c and Figure S2 a-d).

\section{Lysine Acetylation of transcription factors and acetyltransferases/ deacetylases}

1665 human transcription factors (TFs) were download from Human TFDB. In our study, lysine acetylation of 7 TFs (MTA2, LTF, NME2, NME2, SP100, MECP2, STAT3 and STAT1) was significantly upregulated between OSCC and paired healthy tissue, whereas no differentially downregulated acetylated modification happened between OSCC and paired healthy tissue (Table S6). Meanwhile, we calculated lysine acetylation on lysine acetyltransferases (KATs) and lysine deacetylases (KDACs), respectively. More lysine acetylation sites were identified on KATs than on KDACs (Table S7). 11 acetylation sites on EP300 were identified, whereas no significant difference was found between OSCC and paired healthy tissue. In contrast, only lysine 220 of HDAC1 among KDACs was identified and happened significant upregulation.

\section{Discussion}

Lysine acetylation is a major regulator of gene transcription, which is closely associated with OSCC pathology. In our study, we mapped acetylated modification of OSCC and adjacent normal tissues using LC-MS/MS. We identified 8388 acetylation sites in 2706 proteins. 282 upregulated Kac sites in 234 
proteins and 235 downregulated Kac sites in 162 proteins between OSCC tissues and paired adjacent normal tissues were found. Among the upregulated DAPs, the biological process of GO analysis was closely related to cellular response to regulation of apoptotic process, and regulation of programmed cell death related to tumor development $[18,19]$. KEGG enrichment analysis was associated with HIF-1 signaling pathway, ferroptosis, and JAK-STAT signaling pathway. When a response to hypoxia, HIF-1 transcriptional activity is activated by HIF-1a and further promotes tumor invasion and metastasis in head and neck squamous cell carcinoma[20,21]. Ferroptosis is currently considered an effective strategy for overcoming resistance in refractory tumors and may contribute to tumor growth suppression[22]. Activation of JAK-STAT signaling pathway is involved in tumor cell recognition and tumor-driven immune escape[23]. In our study, the upregulated acetylation highly enriched in those pathways, which means acetylated modification may affect the invasion or the response to therapy of OSCC via those processes.

RNA splicing is vital for an essential process that governs many aspects of cellular proliferation, survival, and differentiation. Increasing evidence favors the close relationship between dysregulated RNA splicing and tumorigenesis[24]. Splicing-based therapies for cancer treatment is considered as having enormous potential. PTMs such as ubiquitination, acetylation, and phosphorylation of splicing factors are well studied[25, 26]. In our data, PPI network of the upregulated DAPs involved 228 nodes with 530 edges. 17 proteins in MCODE1 are all among the top 30 hub genes. Biological process of GO analysis of these proteins was associated with mRNA splicing and SRP-dependent cotranslational protein targeting to membrane. The KEGG analysis result was highly enriched in spliceosome and ribosome pathway. Seven proteins (SRSF1, HNPNPM, PRPF8, DHX9, DHX15, RBMX, SNRPG) in MCODE1 were involved in mRNA splicing process and spliceosome pathway. SRSF1 is identified as a proto-oncogene that regulates splicing of important cancer-related genes to promote tumorigenesis[27]. The activity of hnRNPM may reduce by P300-induced upregulated acetylation of hnRNPM although the exactly acetylated site on hnRNPM is unknown[28]. PRPF8, the largest known protein of the spliceosome, plays as the seed gene in the MCODE1 cluster and has been associated with human cancers[29]. DHX9, as well as RBMX, is reported as playing an important role in multiple human malignancies[30, 31]. In our experiments, significantly different acetylated modifications were identified in these hub proteins. HDACs is considered as having great anticancer potential to OSCC. It is reported that there is a potential link between HDACs and RNA splicing that HDACs interact with ribonucleoprotein complexes and spliceosomal, actively control the acetylation states of splicing factors, and further modulate splicing[32]. The deacetylation of the splicing factor SRSF5 results in proteasomal degradation of SRSF5, whose hyperacetylation and upregulation correlates with tumor progression of human lung cancers[25]. Therefore, we hypothesis that acetylated modification may affect the genesis and development of OSCC by regulating the RNA splicing process, especially influencing acetylation of 6 proteins (RPS15A, RPL11, RPS3, RPL24, RPS11, RPL19. Of these, RPS3, RPL24 and RPL19).

In our study, KEGG analysis of MCODE1 also focused on ribosome pathway, including 6 upregulated Kac modified proteins of RPS15A, RPL11, RPS11, RPS3, RPL24, RPL19. Ribosomes are essential for protein production and thus for cellular survival, growth, and proliferation. Ribosome biogenesis includes the synthesis and processing of ribosomal RNAs, assembly of ribosomal proteins, transport to the cytoplasm, 
and binding of ribosomal subunits. Any disruption of the processed above has a close relationship with cancer and other age-related degenerative diseases[33]. RPS15A is a potentially oncogenic gene contributing to colorectal carcinogenesis[34]. PRL11 influents the activity of P53, and further involves in the procession of tumorigenesis [35]. It is also reported that PTMs may regulate RPL11 localization and function[36]. A high expression level of RPS11 is correlated to the poor prognosis of glioblastoma and hepatocellular carcinoma[37, 38]. In these proteins of MCDOE1 involving in ribosome pathway, lower expression of RPS3, RPL19, and RPL24 were related to the overall survival of OSCC. RPS3 inhibits NF-KB pathway activation and then participates in the process of Vitamin D promoting the cisplatin sensitivity of OSCC[39]. The expression level of RPS3 is downregulated by ubiquitylated modification and subsequently results in the inhibition of apoptosis signaling in glioblastoma[40]. Upregulation of RPL19 may result in increased sensitivity to ER stress and enhances cell death in breast cancer cells[41]. Increased K27 acetylation on RPL24 induced by HDACi may result in the recruitment of elF6 to $60 \mathrm{~S}$ subunits and impairing 40S-60S joining and further significantly impair human breast cancer cell viability[42]. Remarkably, K2 and K27 acetylation on RPL24 were identified in our data and K27 happened significantly upregulated acetylation between OSCC and adjacent normal tissues. Thus, we hypothesis that acetylation might regulate ribosome process via upregulating lysine acetylation of 6 hub proteins (RPS15A, RPL11, RPS11, RPS3, RPL24, RPL19) and further affect the genesis and development of OSCC.

The PPI network of the 162 downregulated DAPs included 159 nodes with 94 edges. 11 proteins of the top 30 hub genes had more than 10 acetylation sites. Among the top 30 hub genes, low expression of EIF4A2, RPL12, MYBPC1, RPS6, ARCN1, and TMEM9 were related with poor prognosis in survival analysis, particularly that EIF4A2 and RPL12 with a better survival curve. EIF4A2 is crucial for mRNA binding to ribosome and plays an important role in translation initiation. It is reported EIF4A2 promotes colorectal cancer experimental metastasis and affected the efficacy of antitumor drugs[43].

Phosphorylation of RPL12/UL11 regulates elongation through interaction with two elongation GTPases, eEF1A and eEF2, and further regulates mitotic translation[44].The phenomenon supports the possibility that core ribosomal proteins can be modified by many types of PTMs. To our knowledge, this is the first study to investigate lysine acetylation on proteins involved in ribosome pathway, particularly the ones that acted as hub genes and related with survival of OSCC, which may be a potential therapeutic direction of OSCC in the future.

\section{Conclusion}

In summary, our study mapped an overview of acetylation in OSCC and clarified the differently acetylated modification sites in OSCC and adjacent normal tissues. We provide a bioinformatic analysis of differently Kac modified proteins. To our knowledge, this is the first study to investigate lysine acetylation on proteins involved in ribosome pathway, particularly the ones that acted as hub genes and related to the OSCC survival. Our data may provide new insight into the pathogenesis of OSCC and even propose a potential therapeutic direction of OSCC in the future. 


\section{Declarations}

Acknowledgements

Not applicable.

\section{Authors' contributions}

Jingjing Dong and Jingquan He contributed to the writing of the original draft and preparation; Lianghong Yin, Donge Tang and Yong Dai contributed to the conceptualization; Zeyu Zhang, Wei Zhang and Yixi Li contributed to the methodology; Wenxin Zuo, Jianming Tang, Liusheng Lai, and Manhua Yun participated in the collection of tissue samples and clinical data. Zhipeng Zeng and Wanxia Cai contributed to the project administration. Hongliang Xie contributed to the preparation of the manuscript. The authors are grateful especially the trial participants and their families.

\section{Funding}

This work was supported by the Science and Technology Plan of Shenzhen (grant numbers, JCYJ20190807145815129), Guangxi Key Laboratory of Metabolic Diseases Research (grant numbers, 20-065-76), the Science and Technology Plan of Shenzhen (grant numbers, JCYJ20180306140810282), the Natural Science Foundation of Guangxi (grant numbers, 2017GXNSFAA198185), the Guangdong Province Union Training Postgraduate Demonstration Base (grant numbers, 20190630).

\section{Availability of data and materials}

The datasets supporting the conclusions of the current study are available from the corresponding author on reasonable request. Please contact corresponding author, if you want to request the dataset.

\section{Ethics approval and consent to participate}

This study included human tissues and data. The research protocol was approved by the Clinical Research Ethics Committee of the Shenzhen People's Hospital.

\section{Consent for publication}

Not applicable.

\section{Competing interests}

The authors declare that they have no known competing interests.

\section{Abbreviations}

Kac Lysine acetylation 
OSCC Oral squamous cell carcinoma

LC-MS/MS Liquid chromatography-tandem mass spectrometry

DAPs Different acetylation proteins

PTMs Posttranslational modification

COG Clusters of Orthologous Groups

KOG EuKaryotic Orthologous Groups

GO Gene Ontology

KEGG Kyoto Encyclopedia of Genes and Genomes

DEPs Differential expression proteins

TFs Transcription factors

KATs Lysine acetyltransferases

KDACs Lysine deacetylases

HDAC Histone deacetylase

MCODE Molecular Complex Detection

UCSC University of California Santa Cruz

\section{References}

1. Carnielli CM, Macedo CCS, De Rossi T, Granato DC, Rivera C, Domingues RR, et al. Combining discovery and targeted proteomics reveals a prognostic signature in oral cancer. Nat Commun. 2018;9(1):3598.

2. da Silva SD, Hier M, Mlynarek A, Kowalski LP, Alaoui-Jamali MA. Recurrent oral cancer: current and emerging therapeutic approaches. Front Pharmacol. 2012;3:149.

3. Minguez P, Parca L, Diella F, Mende DR, Kumar R, Helmer-Citterich M, et al. Deciphering a global network of functionally associated post-translational modifications. Mol Syst Biol. 2012;8:599.

4. Zhou T, Xia X, Liu J, Wang G, Guo Y, Guo X, et al. Beyond single modification: Reanalysis of the acetylproteome of human sperm reveals widespread multiple modifications. J Proteomics. 2015;126:296-302.

5. Samanta L, Swain N, Ayaz A, Venugopal V, Agarwal A. Post-Translational Modifications in sperm Proteome: The Chemistry of Proteome diversifications in the Pathophysiology of male factor 
infertility. Biochim Biophys Acta. 2016;1860(7):1450-65.

6. Wan J, Liu H, Chu J, Zhang H. Functions and mechanisms of lysine crotonylation. J Cell Mol Med. 2019;23(11):7163-9.

7. Han ZJ, Feng YH, Gu BH, Li YM, Chen H. The post-translational modification, SUMOylation, and cancer (Review). Int J Oncol. 2018;52(4):1081-94.

8. Gil J, Ramírez-Torres A, Encarnación-Guevara S. Lysine acetylation and cancer: A proteomics perspective. J Proteomics. 2017;150:297-309.

9. Shimko JC, North JA, Bruns AN, Poirier M, G,Ottesen JJ. Preparation of fully synthetic histone H3 reveals that acetyl-lysine 56 facilitates protein binding within nucleosomes. $\mathrm{J}$ Mol Biol. 2011;408(2):187-204.

10. He L, Gao L, Shay C, Lang L, Lv F, Teng Y. Histone deacetylase inhibitors suppress aggressiveness of head and neck squamous cell carcinoma via histone acetylation-independent blockade of the EGFRArf1 axis. J Exp Clin Cancer Res. 2019;38(1):84.

11. Sugita K, Koizumi K, Yoshida H. Morphological reversion of sis-transformed NIH3T3 cells by trichostatin A. Cancer Res. 1992;52(1):168-72.

12. Chen F, Qi S, Zhang X, Wu J, Yang X, Wang R. IncRNA PLAC2 activated by H3K27 acetylation promotes cell proliferation and invasion via the activation of Wnt/ $\beta$-catenin pathway in oral squamous cell carcinoma. Int J Oncol. 2019;54(4):1183-94.

13. Giudice FS, Pinto DS Jr, Nör JE, Squarize CH, Castilho RM. Inhibition of histone deacetylase impacts cancer stem cells and induces epithelial-mesenchyme transition of head and neck cancer. PLoS One. 2013;8(3):e58672.

14. Zhang H, Han W. Protein Post-translational Modifications in Head and Neck Cancer. Front Oncol. 2020;10:571944.

15. Dong J, Li Y, Zheng F, Chen W, Huang S, Zhou X, et al. Co-occurrence of Protein Crotonylation and 2Hydroxyisobutyrylation in the Proteome of End-Stage Renal Disease. ACS Omega. 2021;6(24):15782-93.

16. Hebert AS, Dittenhafer-Reed KE, Yu W, Bailey DJ, Selen ES, Boersma MD, et al. Calorie restriction and SIRT3 trigger global reprogramming of the mitochondrial protein acetylome. Mol Cell. 2013;49(1):186-99.

17. Chai X, Guo J, Dong R, Yang X, Deng C, Wei C, et al. Quantitative acetylome analysis reveals histone modifications that may predict prognosis in hepatitis B-related hepatocellular carcinoma. Clin Transl Med. 2021;11(3):e313.

18. Yang $S$, Mao Y, Zhang H, Xu Y, An J, Huang Z. The chemical biology of apoptosis: Revisited after 17 years. Eur J Med Chem. 2019;177:63-75.

19. Kesavardhana S, Malireddi RKS, Kanneganti TD. Caspases in Cell Death, Inflammation, and Pyroptosis. Annu Rev Immunol. 2020;38:567-95. 
20. Zhu G, Peng F, Gong W, She L, Wei M, Tan H, et al. Hypoxia promotes migration/invasion and glycolysis in head and neck squamous cell carcinoma via an HIF-1a-MTDH loop. Oncol Rep. 2017;38(5):2893-900.

21. Bu M, Liu X, Liu X, Xu W. Upregulation of fascin-1 is involved in HIF-1a-dependent invasion and migration of hypopharyngeal squamous cell carcinoma. Int J Oncol. 2019;55(2):488-98.

22. Li S, Liu Y, Li J, Zhao X, Yu D. Mechanisms of Ferroptosis and Application to Head and Neck Squamous Cell Carcinoma Treatments. DNA Cell Biol. 2021;40(6):720-32.

23. Owen KL, Brockwell NK, Parker BS. JAK-STAT Signaling: A Double-Edged Sword of Immune Regulation and Cancer Progression. Cancers (Basel). 2019; 11(12).

24. Wang E, Aifantis I. RNA Splicing and Cancer. Trends Cancer. 2020;6(8):631-44.

25. Chen $Y$, Huang Q, Liu W, Zhu Q, Cui CP, Xu L, et al. Mutually exclusive acetylation and ubiquitylation of the splicing factor SRSF5 control tumor growth. Nat Commun. 2018;9(1):2464.

26. Xu L, Zhang H, Mei M, Du C, Huang X, Li J, et al. Phosphorylation of serine/arginine-rich splicing factor 1 at tyrosine 19 promotes cell proliferation in pediatric acute lymphoblastic leukemia. Cancer Sci. 2018;109(12):3805-15.

27. Lv Y, Zhang W, Zhao J, Sun B, Qi Y, Ji H, et al. SRSF1 inhibits autophagy through regulating Bcl-x splicing and interacting with PIK3C3 in lung cancer. Signal Transduct Target Ther. 2021;6(1):108.

28. Siam A, Baker M, Amit L, Regev G, Rabner A, Najar RA, et al. Regulation of alternative splicing by p300-mediated acetylation of splicing factors. Rna. 2019;25(7):813-24.

29. Wood KA, Eadsforth MA, Newman W, G,O'Keefe RT. The Role of the U5 snRNP in Genetic Disorders and Cancer. Front Genet. 2021;12:636620.

30. Yan X, Chang J, Sun R, Meng X, Wang W, Zeng L, et al. DHX9 inhibits epithelial-mesenchymal transition in human lung adenocarcinoma cells by regulating STAT3. Am J Transl Res. 2019;11(8):4881-94.

31. Song Y, He S, Ma X, Zhang M, Zhuang J, Wang G, et al. RBMX contributes to hepatocellular carcinoma progression and sorafenib resistance by specifically binding and stabilizing BLACAT1. Am J Cancer Res. 2020;10(11):3644-65.

32. Rahhal R, Seto E. Emerging roles of histone modifications and HDACs in RNA splicing. Nucleic Acids Res. 2019;47(10):4911-26.

33. Turi Z, Lacey M, Mistrik M, Moudry P. Impaired ribosome biogenesis: mechanisms and relevance to cancer and aging. Aging. 2019;11(8):2512-40.

34. Wan BS, Wang XY, Tiang J, Zhou C, Lin J, Wang Z. Ribosomal protein RPS15A augments proliferation of colorectal cancer RKO cells via regulation of BIRC3, p38 MAPK and Chk1. Eur Rev Med Pharmacol Sci. 2021;25(11):3967-80.

35. Kayama K, Watanabe S, Takafuji T, Tsuji T, Hironaka K, Matsumoto M, et al. GRWD1 negatively regulates $\mathrm{p} 53$ via the RPL11-MDM2 pathway and promotes tumorigenesis. EMBO Rep. 2017;18(1):123-37. 
36. El Motiam A, Vidal S, de la Cruz-Herrera CF, Da Silva-Álvarez S, Baz-Martínez M, Seoane R, et al. Interplay between SUMOylation and NEDDylation regulates RPL11 localization and function. Faseb j. 2019;33(1):643-51.

37. Zhou C, Sun J, Zheng Z, Weng J, Atyah M, Zhou Q, et al. High RPS11 level in hepatocellular carcinoma associates with poor prognosis after curative resection. Ann Transl Med. 2020;8(7):466.

38. Yong WH, Shabihkhani M, Telesca D, Yang S, Tso JL, Menjivar JC, et al. Ribosomal Proteins RPS11 and RPS20, Two Stress-Response Markers of Glioblastoma Stem Cells, Are Novel Predictors of Poor Prognosis in Glioblastoma Patients. PLoS One. 2015;10(10):e0141334.

39. Huang Z, Zhang Y, Li H, Zhou Y, Zhang Q, Chen R, et al. Vitamin D promotes the cisplatin sensitivity of oral squamous cell carcinoma by inhibiting LCN2-modulated NF-KB pathway activation through RPS3. Cell Death Dis. 2019;10(12):936.

40. Kim W, Youn H, Lee S, Kim E, Kim D, Sub Lee J, et al. RNF138-mediated ubiquitination of rpS3 is required for resistance of glioblastoma cells to radiation-induced apoptosis. Exp Mol Med. 2018;50(1):e434.

41. Hong M, Kim H, Kim I. Ribosomal protein L19 overexpression activates the unfolded protein response and sensitizes MCF7 breast cancer cells to endoplasmic reticulum stress-induced cell death. Biochem Biophys Res Commun. 2014;450(1):673-8.

42. Wilson-Edell KA, Kehasse A, Scott GK, Yau C, Rothschild DE, Schilling B, et al. RPL24: a potential therapeutic target whose depletion or acetylation inhibits polysome assembly and cancer cell growth. Oncotarget. 2014;5(13):5165-76.

43. Chen ZH, Qi JJ, Wu QN, Lu JH, Liu ZX, Wang Y, et al. Eukaryotic initiation factor 4A2 promotes experimental metastasis and oxaliplatin resistance in colorectal cancer. J Exp Clin Cancer Res. 2019;38(1):196.

44. Imami K, Milek M, Bogdanow B, Yasuda T, Kastelic N, Zauber $\mathrm{H}$, et al. Phosphorylation of the Ribosomal Protein RPL12/uL11 Affects Translation during Mitosis. Mol Cell. 2018;72(1):84-98.e9.

\section{Figures}


a

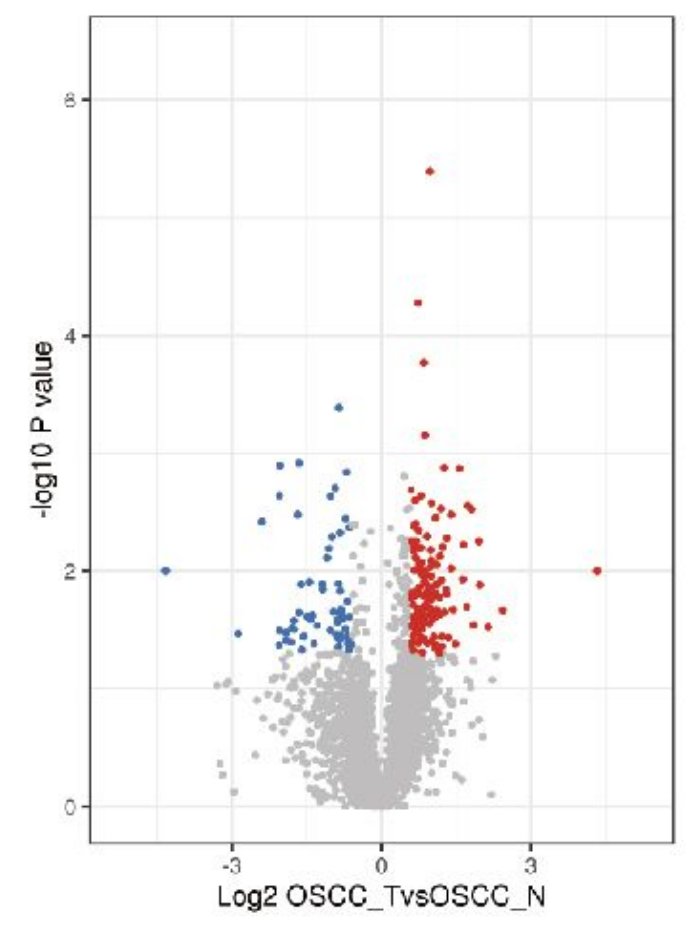

C DEPs

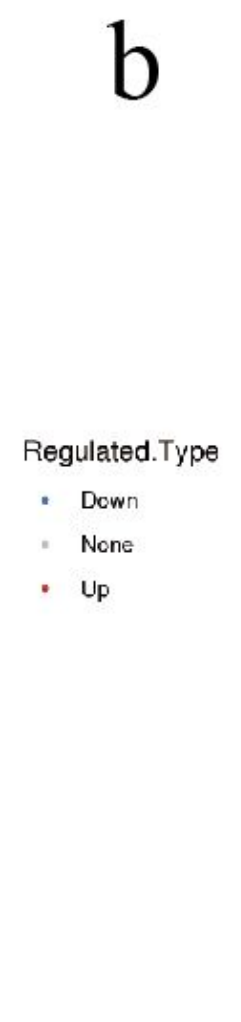

DAPs

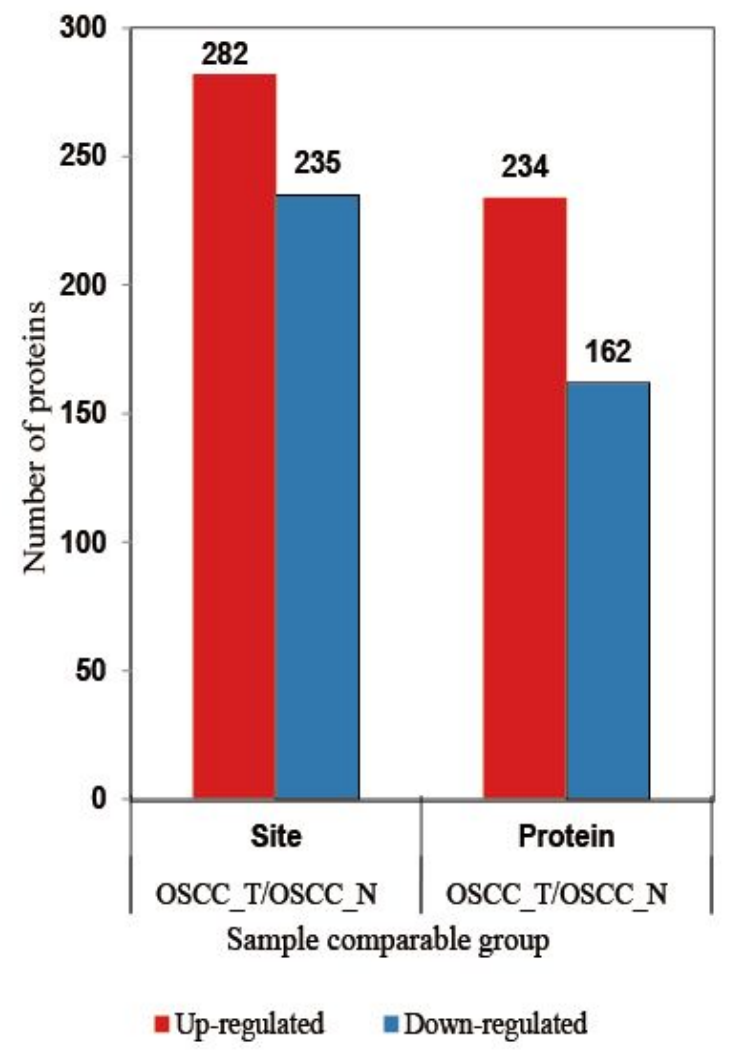

\section{Figure 1}

Qualitative analysis of lysine acetylation between OSCC and adjacent normal tissues. (a) The volcano plot of the different acetylation. (b) Histogram of quantity distribution of differential expression proteins (DEPs) and differentially acetylated proteins (DAPs). (c) Venn graph representing the overlap of DEPs and DAPs. 

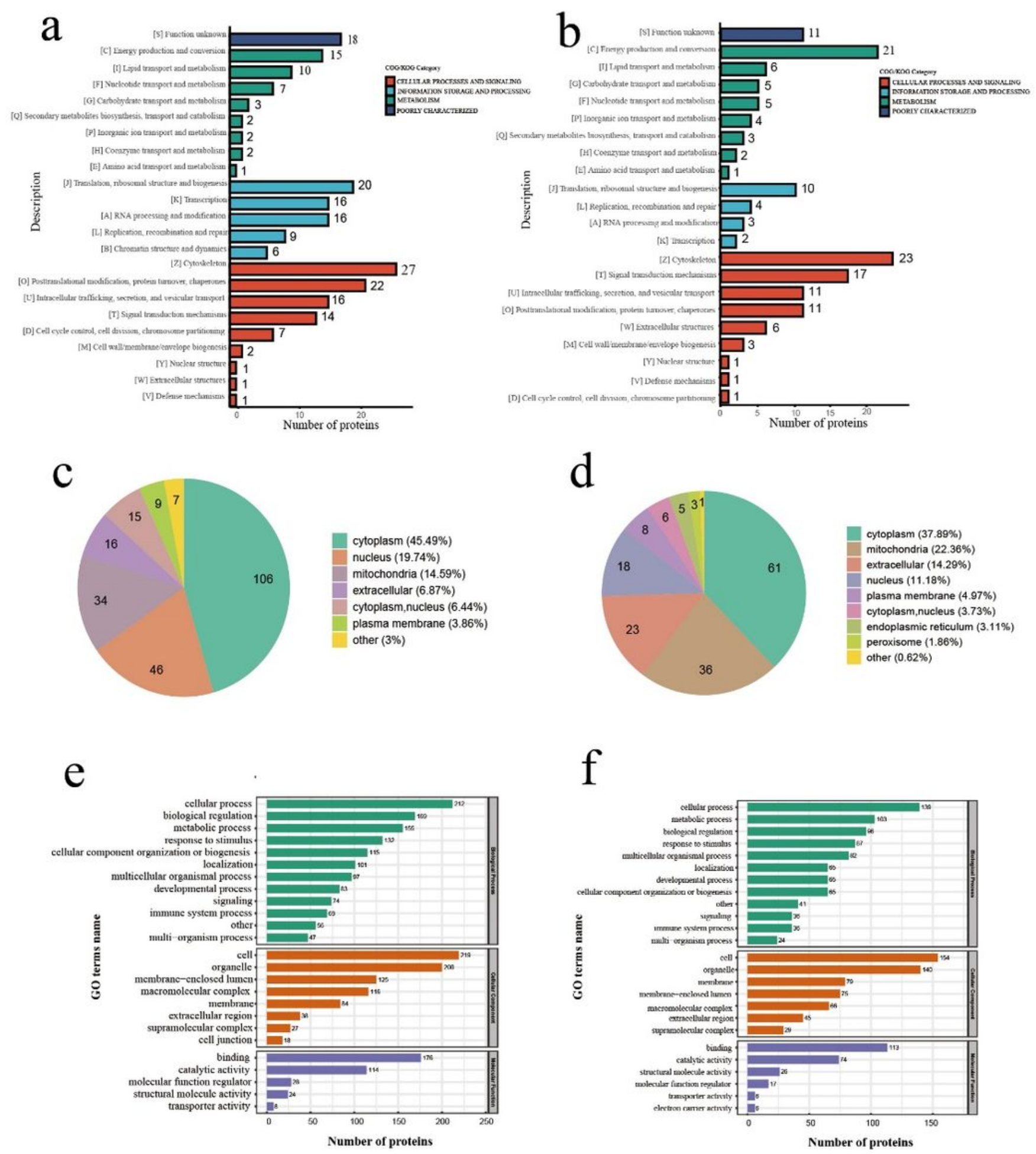

\section{Figure 2}

Functional characterization of differentially lysine acetylation proteins between OSCC and adjacent normal tissues. $(a, b)$ The upregulated/downregulated acetylated protein distribution in COG category distribution. (c, d) Subcellular localization of upregulated/downregulated acetylated protein. (e, f) Gene Ontology annotation of upregulated/downregulated acetylated protein. 


\section{a}

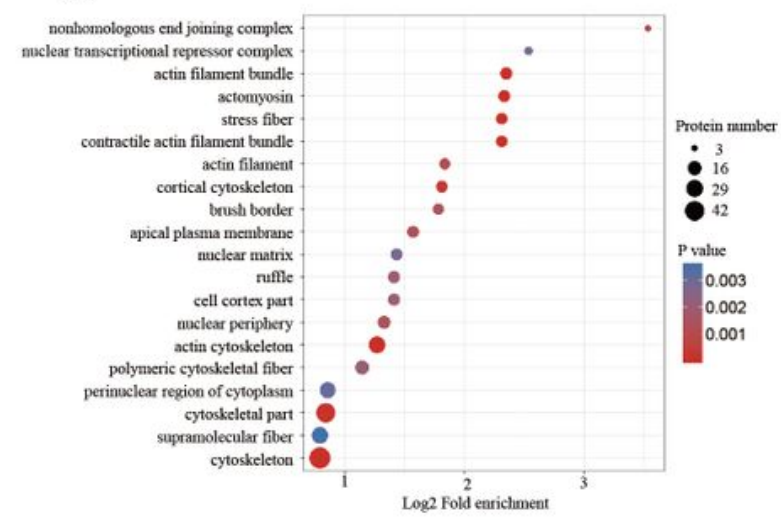

C

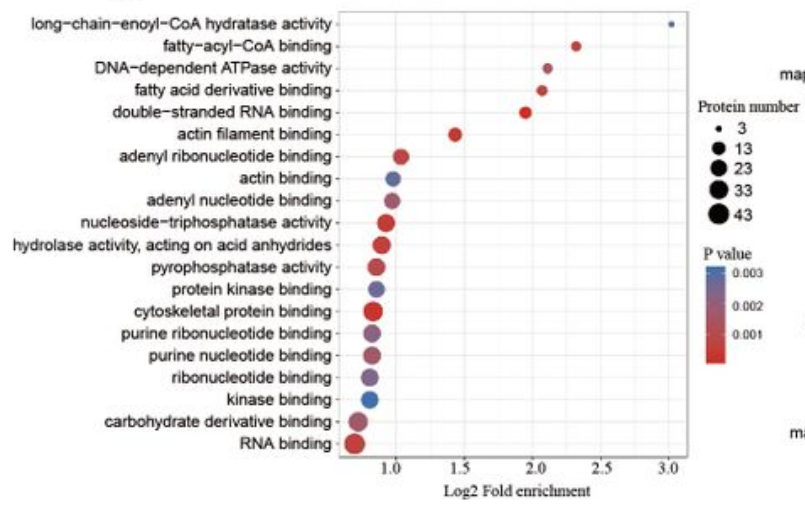

b

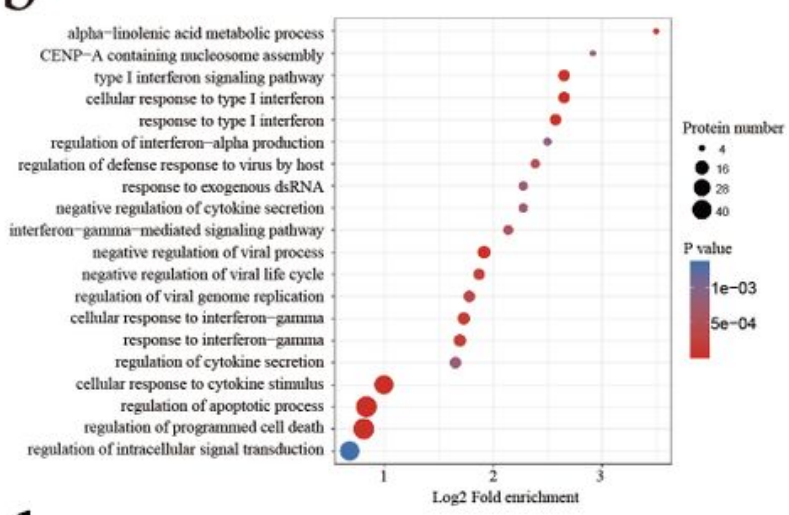

d

map00120 Primary bile acid biosynthesis map00592 alpha-linolenic acid isyethesis 01040 Biosynthesis of unsentuted fatty acids. map03450 Non-homologous end-7oining. map03450 Non-homologous end-joining
map05160 Hepatitis C map04630 JAK-STAT signa Hepatitis C map01212 Fatty acid metabolism

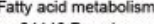
map04146 Peroxisome map04216 Ferroptosis mapeosip Pryste measlos. map00620 Pyruvate metabolism map 132 sax vins 1 infection map05132 Salmonella infection map05100 Bacterial invasion of epithelial cells mapo0071 Fap04110 Cell cycle mapo0071 Faty acid degradation map00640 Propanoate metabolism map04066 HIF-1 signaling patthway map04621 NOD-like receptor signaling pathway map05205 Proteoglycans in cancer

\section{e}

Guanylate-binding protein, N-terminal domain 2.53

Guanylate-binding protein, C-terminal domain

Ku70/Ku80 N-terminal alpha/beta domain Ku70/Ku80 beta-barrel domain 2.07 2'-5'-oligoadenylate synthetase 1, domain 2, C-terminus 2.07

Thiolase, N-terminal domain 1.9

Thiolase, C-terminal domain 1.9

Thioesterase superfamily 1.62

STAT protein, protein interaction domain 1.62

STAT protein, DNA binding domain 1.62

RecF/RecN/SMC N terminal domain 1.62

Oligonucleotide/oligosaccharide-binding (OB)-fold 1.62

NOPS (NUC059) domain 1.62

$\mathrm{MCM} 2 / 3 / 5$ family

IMP dehydrogenase / GMP reductase domain 1.62

Helicase associated domain (HA2) 1.62

Filamin/ABP280 repeat 1.62

Elongation factor Tu domain 2

Acyl-CoA dehydrogenase, middle domain 1.36

\section{$-\log 10$ (Fisher' exact test $p$ value)}

\section{Figure 3}

Functional enrichment analysis of upregulated lysine acetylation proteins between OSCC and adjacent normal tissues. (a) Cellular component in the $G O$ enrichment analysis. (b) Biological process in the $G O$ enrichment analysis. (c) Molecular function in the GO enrichment analysis. (d) KEGG analysis of upregulated lysine acetylation proteins. 
a

b
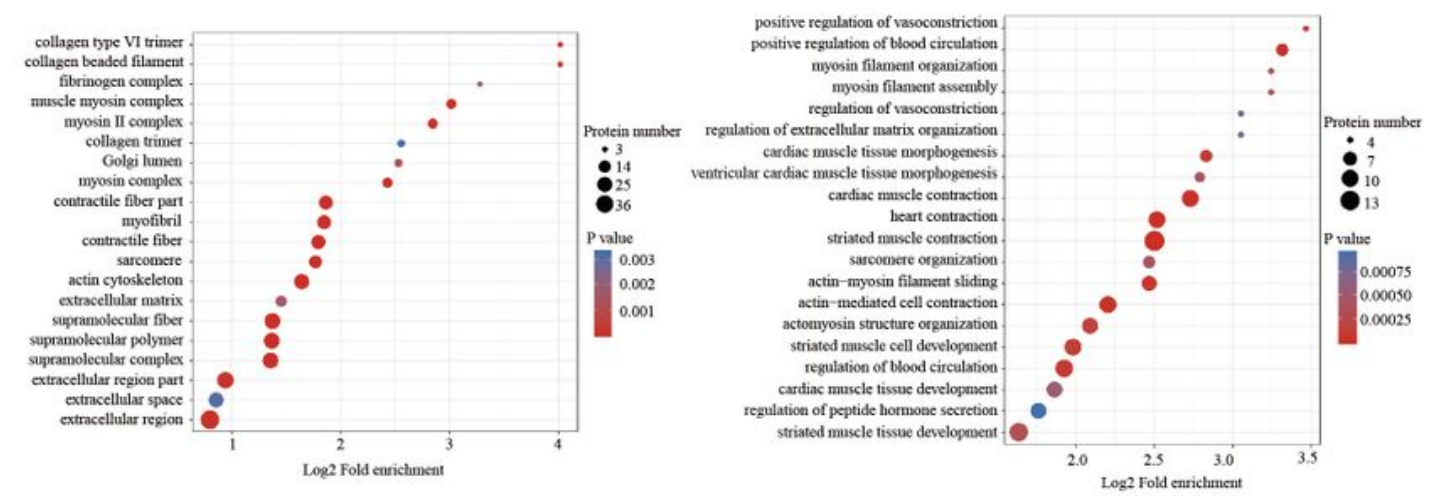

c

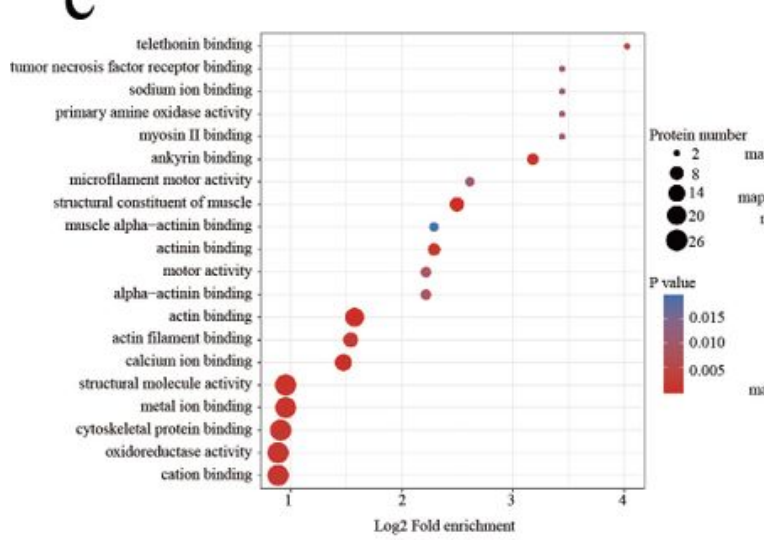

d

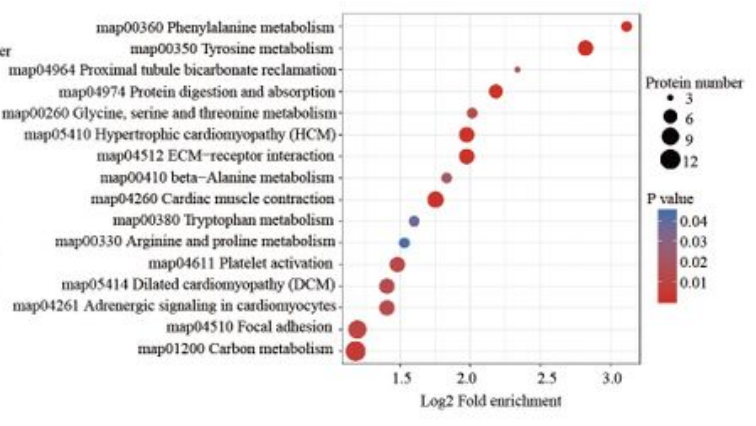

e

$\mathrm{Ca} 2+$ insensitive $\mathrm{EF}$ hand 3.46

Myosin N-terminal SH3-like domain Calponin homology $(\mathrm{CH})$ domain

Filamin/ABP280 repeat
Spectrin repeat Spectrin repeat Domain of unknown function (DUF4009) Guanylate-binding protein, N-terminal domain PDZ domain (Also known as DHR or GLGF) Myosin head (motor domain) Vinculin Binding Site Talin, middle domain Ku70/Ku80 N-terminal alpha/beta domain Ku70/Ku80 beta-barrel domain Ferl (NUC094) domain

FerB (NUC096) domain

EF-hand domain pair

2'-5'-oligoadenylate synthetase 1 , domain 2, C-terminus

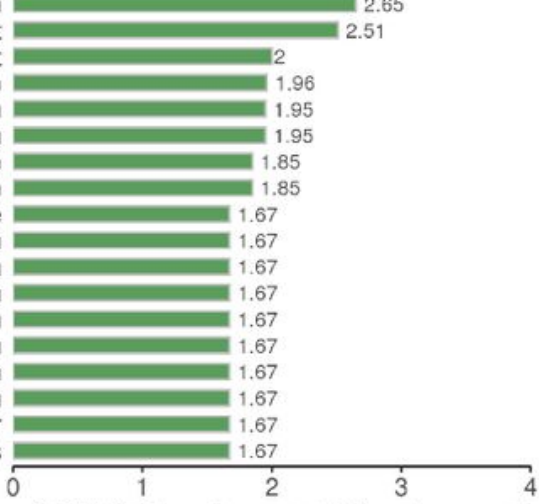

-log10(Fisher' exact test $p$ value)

\section{Figure 4}

Functional enrichment analysis of downregulated lysine acetylation proteins between OSCC and adjacent normal tissues. (a) Cellular component in the $\mathrm{GO}$ enrichment analysis. (b) Biological process in the GO enrichment analysis. (c) Molecular function in the GO enrichment analysis. (d) KEGG analysis of downregulated lysine acetylation proteins. (e) Column graphs show the domain enrichment. 


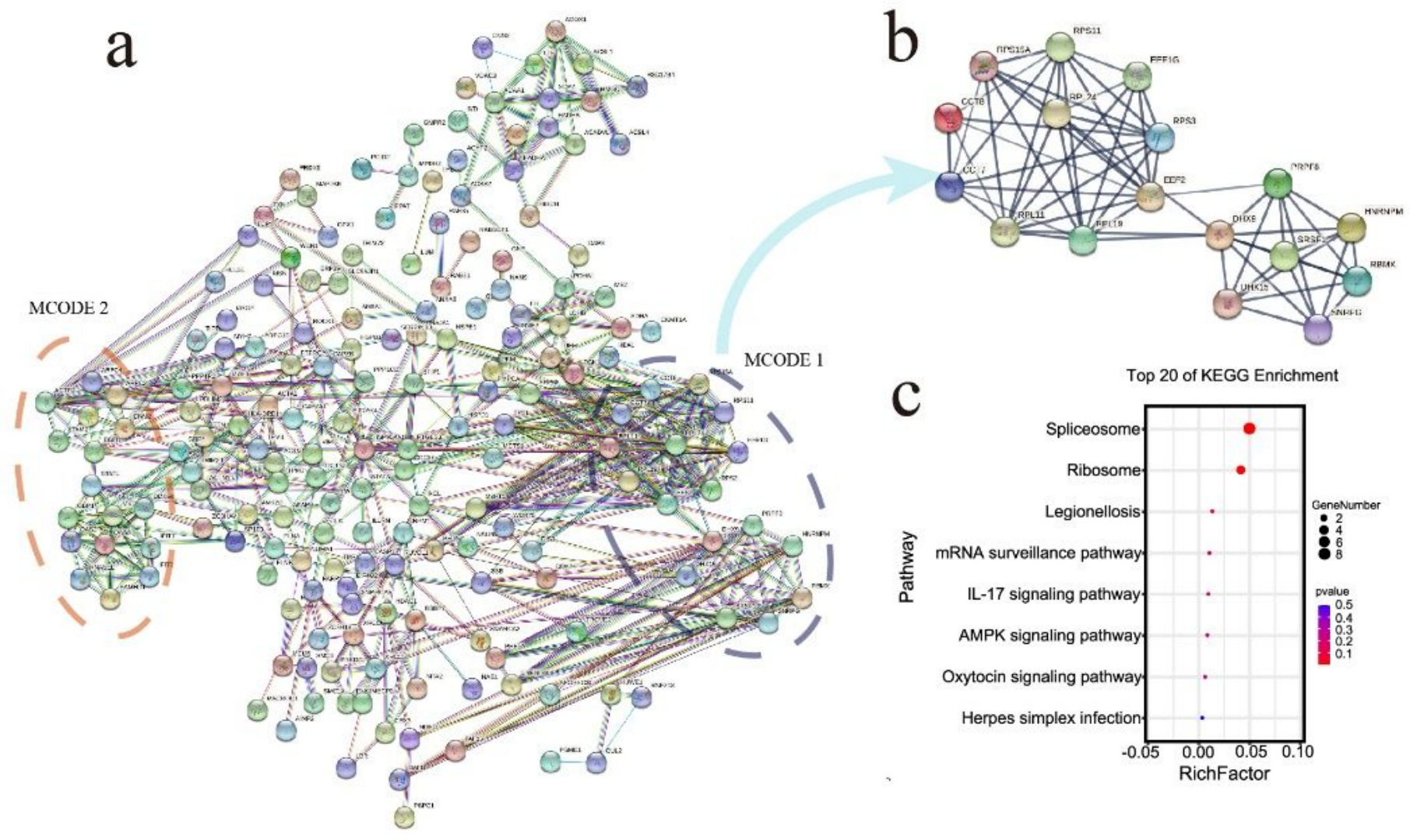

Figure 5

PPI network analysis of upregulated lysine acetylation proteins and functional enrichment analysis of proteins in MCODE1. (a) PPI network analysis of upregulated lysine acetylation proteins between OSCC and adjacent normal tissues was performed. Two most significant modules were yielded with MCODE. (b) 17 proteins in MCODE 1((MCODE score 9.2). (c) GO enrichment analysis of MCODE 1. (d) KEGG analysis of MCODE 1. 
a
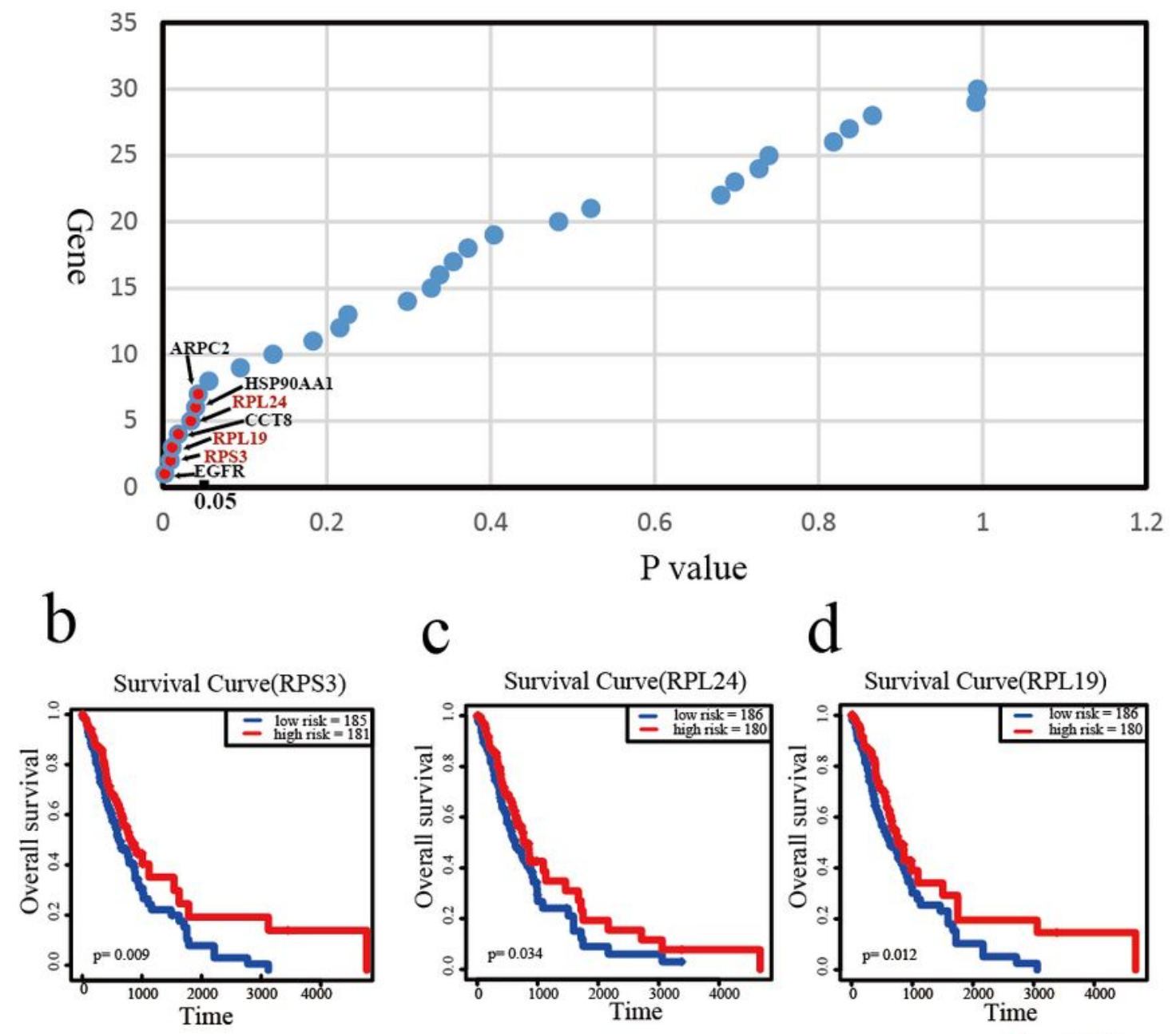

C

d
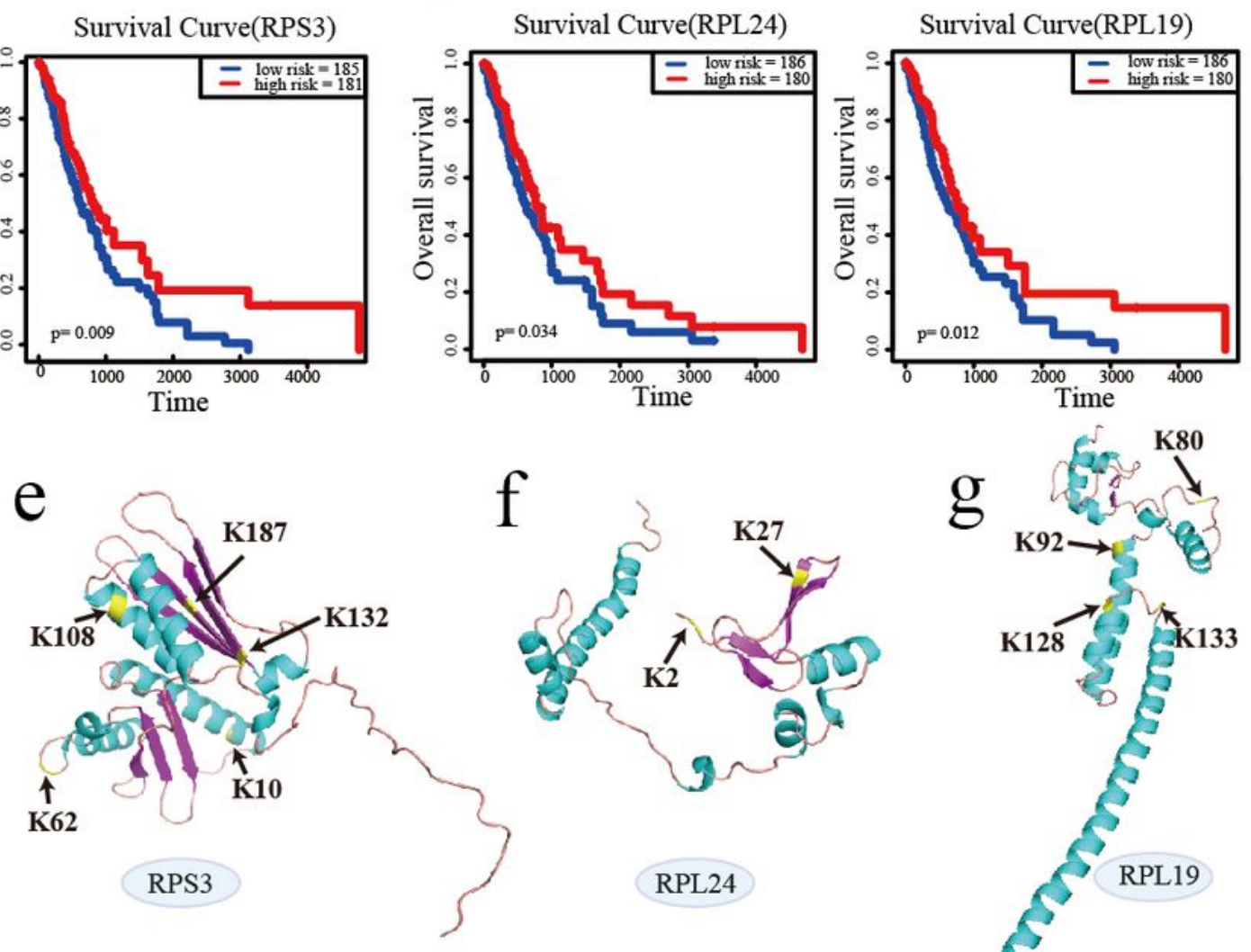

RPL24

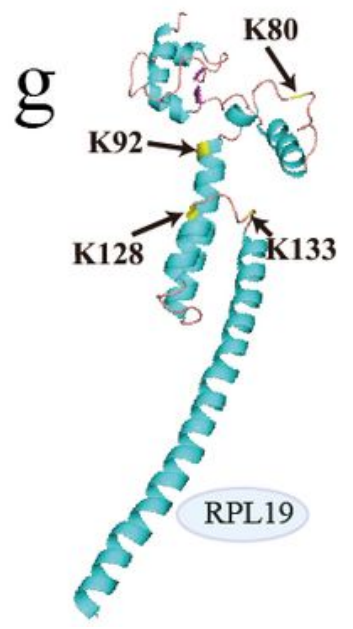

\section{Figure 6}

The top 30 hub gene of upregulated acetylated proteins and Survival Analysis. (a) Scatter plot charts of the top 30 hub gene were shown. The proteins with $P$ value $<0.05$ in survival Analysis were annotated by red points, particularly three proteins involved in MCODE1 were marked in red font. (b, c, d) survival curve of RPS3, RPL24, RPL19 from MCODE1, respectively. $(e, f, g)$ Three-dimensional structure of RPS3, RPL24, RPL19 constructed using SWISS MODEL, respectively. Acetylation sites were labeled yellow. 

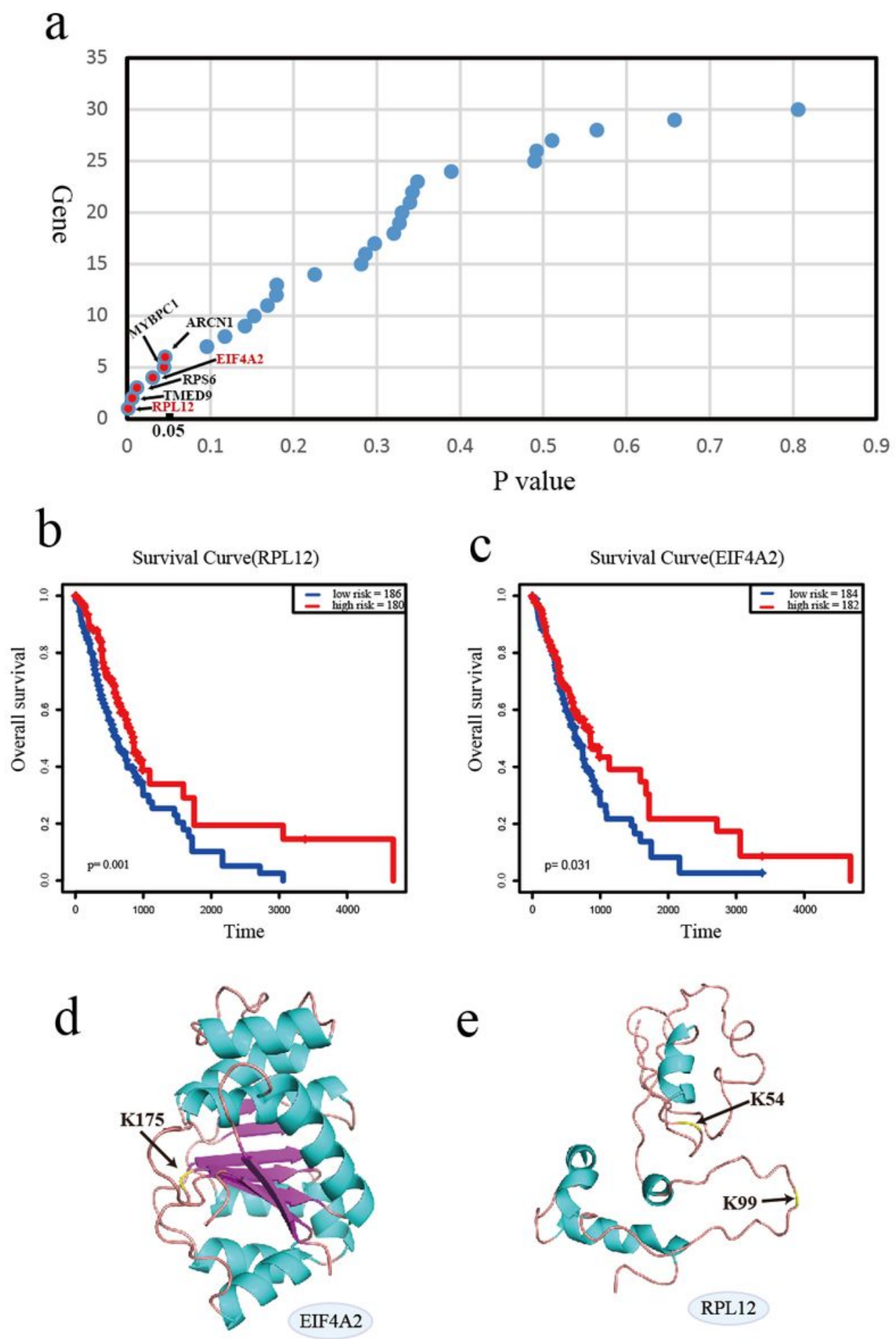

\section{Figure 7}

The top 30 hub gene of downregulated acetylated proteins and Survival Analysis. (a) Scatter plot charts of the top 30 hub gene were shown. The proteins with $P$ value $<0.05$ in survival Analysis were annotated by red points. (b, c) RPL12 and EIF4A2 with better survival curve were shown, respectively. (d, e) Threedimensional structure of RPL12 and EIF4A2 constructed using SWISS MODEL, respectively. Acetylation sites were labeled yellow. 


\section{Supplementary Files}

This is a list of supplementary files associated with this preprint. Click to download.

- SupplementaryMaterial.doc 